\title{
КОМПОЗИЦИЯ ГЕНЕРАТОРОВ НЕЧЕТКИХ ОПЕРАЦИЙ
}

\author{
Т. М. Леденева \\ Воронежский государственный университет
}

Поступила в редакцию 15.09.2019 г.

\begin{abstract}
Аннотация. Данная статья посвящена исследованию аддитивных генераторов в форме дробно-линейных функций. Показано, что композиции возрастающих и/или убывающих генераторов также являются генераторами. Для каждого случая найдены соответствующие треугольные нормы или конормы. Установлено, что треугольные нормы и конормы, полученные на основе дробно-линейных функций, а также двойственные им имеют одинаковую структуру.
\end{abstract}

Ключевые слова: треугольные нормы и конормы, убывающие и возрастающие генераторы.

\section{ВВЕДЕНИЕ}

Одной из основных проблем нечеткого моделирования является формализация семантических связок «и» и «или». Предложенные первоначально Л. Заде операции $\min$ и max по-прежнему часто используются в современных разработках по нечетким прикладным системам, поскольку их физических смысл вполне очевиден, а алгебра, построенная на основе этих операций, с математической точки зрения имеет хорошие характеристики. Важнейшим достижением является аксиоматический подход, результатом которого стало появления треугольных норм и конорм [1-3]. Они возникли как естественное обобщение классического «неравенства треугольника» при рассмотрении вероятностных метрических пространств [4]. Дальнейшее развитие теории треугольных норм и конорм связано с функциональными уравнениями, которые порождают различные представления нечетких операций, в частности, с помощью аддитивных и мультипликативных генераторов. Аддитивный генератор позволяет перейти к аддитивной форме операции, и такое представление связано со свойством ассоциативности бинарных операций. Наиболее известные параметрические семейства треугольных норм и конорм и соответствую-

() Леденева Т. М., 2019 щие им аддитивные генераторы можно найти в $[2,5]$. Некоторые из них представлены в табл. 1. Современные исследования касаются решения ряда проблем [6], связанных с различными обобщениями и модификациями такого представления, в том числе, для специальных классов нечетких операций; с выявлением взаимосвязи свойств аддитивного генератора и алгебраических свойств треугольных норм и конорм; с формированием правил или процедур для восстановления аддитивных генераторов для заданной нечеткой операции.

Заметим, что многочисленный класс нечетких операций составляют те, которые представимы симметричными рациональными функциями вида

$$
F(x, y)=\frac{a_{0}+a_{1}(x+y)+a_{2} x y}{b_{0}+b_{1}(x+y)+b_{2} x y} .
$$

Введем необходимые определения, базируясь на $[1,5]$.

Треугольной нормой называется монотонная, коммутативная и ассоциативная бинарная операция $T:[0,1]^{2} \rightarrow[0,1]$, такая что для любого $x \in X$ выполняется $T(x, 1)=T(1, x)=x$.

Треугольной конормой называется двойственная к треугольной норме $T$ операция $S:[0,1]^{2} \rightarrow[0,1]$, для которой имеют место свойства коммутативности, ассоциативности, монотонности, а также свойство $S(x, 0)=S(0, x)=x$. 
Треугольные нормы и конормы

\begin{tabular}{|c|c|}
\hline$T(x, y)$ & $S(x, y)$ \\
\hline$T_{M}(x, y)=\min (x, y)$ & $S_{M}(x, y)=\max (x, y)$ \\
\hline$T_{P}(x, y)=x y$ & $S_{P}(x, y)=x+y-x y$ \\
\hline$T_{L}(x, y)=\max (0, x+y-1), t_{L}(x)=1-x$ & $S_{L}(x, y)=\min (1, x+y), S_{L}(x)=x$ \\
\hline$T_{0}(x, y)=\frac{x y}{x+y-x y}, t_{0}(x)=\frac{1-x}{x}$ & $S_{-1}(x, y)=\frac{x+y-2 x y}{1-x y}, S_{-1}(x)=\frac{x}{1-x}$ \\
\hline$T_{\alpha}(x, y)=\frac{x y}{\alpha+(1-\alpha)(x+y-x y)}, \alpha>0$ & $S_{\beta}(x, y)=\frac{(\beta-1) x y+x+y}{1+\beta x y}, \beta>-1$ \\
\hline$T_{H}(x, y)=\frac{x y}{h+(1-h)(x+y-x y)}, h>0$ & $S_{H}(x, y)=\frac{x+y+(h-2) x y}{h+(h-1) x y}, h>0$ \\
\hline$T_{\lambda}(x, y)=\max (0, x+y-1-\lambda(1-x)(1-y)), \lambda>-1$ & $S_{\lambda}(x, y)=\min (1, x+y+\lambda x y), \lambda>-1$ \\
\hline$T_{w}(x, y)=\max \left(0, \frac{x+y+w x y-1}{1+w}\right), w>-1$ & $S_{w}(x, y)=\min \left(1, x+y-\frac{w}{1-w} x y\right), w>-1$ \\
\hline
\end{tabular}

Треугольная норма моделирует операции типа умножения (пересечение нечетких множеств, конъюнкция), а конорма - операции типа сложения (объединение нечетких множеств, дизъюнкция). Системы $\langle[0,1], T, \leq\rangle$ и $\langle[0,1], S, \leq\rangle$ являются абелевыми полугруппами с нейтральным элементом 0 и 1 соответственно и отношением порядка $\leq$.

Среди треугольных норм и конорм выделяют класс архимедовых операций.

Норма $T$ называется архимедовой, если для всех $x \in X$ выполняется неравенство $T(x, x)<x$. С учетом двойственности, аналогичное неравенство для треугольной конормы имеет вид $S(x, x)>x$.

Известно, что любую архимедову треугольную норму $T$ можно представить в виде

$$
T(x, y)=t^{(-1)}(t(x)+t(y)),
$$

где $t:[0,1] \rightarrow \mathbb{R}$ - убывающая, непрерывная функция, такая, что $t(1)=0$ и называемая убываюшим генератором.

Для архимедовых треугольных конорм имеет место аналогичное представление

$$
S(x, y)=s^{(-1)}(s(x)+s(y)),
$$

где $s:[0,1] \rightarrow \mathbb{R}$ - непрерывная, возрастающая функция со свойством $s(0)=0$, называемая возрастающим генератором.
В формулах (2) и (3) функции $t^{(-1)}$ и $s^{(-1)}-$ псевдообратные функции.

Убывающий $t$ и возрастающий $s$ генераторы называются аддитивными. Важно, что аддитивные генераторы определяются с точностью до положительной мультипликативной константы, т. е. если функция $\varphi$ является аддитивным генератором, то функция $c \cdot \varphi$ для $c>0$ также является аддитивным генератором [5]. Важно, что константа $c$ не влияет на определение соответствующей треугольной нормы или конормы [2].

Характеристика основных классов аддитивных генераторов - одна из наиболее важных проблем функционального представления нечетких операций [6]. Заметим, что единой процедуры для построения аддитивного генератора заданной треугольной нормы или конормы не существует. Однако для некоторых классов треугольных норм и конорм такие подходы предлагаются (например, $[7,8])$. В $[9,10]$ определены типы аддитивных генераторов для класса треугольных норм и конорм, представимых функциями вида (1), т. е. отношением двух многочленов или, в частном случае, многочленом, в которые переменные входят не выше, чем в первой степени. В [11] исследуются генераторы в 


\section{Т. М. Леденева}

форме дробно-линейных функций - LFAG. Определены ограничения, при выполнении которых дробно-линейная функция общего вида является убывающим или возрастающим $L F A G$-генератором. Предложен новый класс треугольных норм и конорм, который обобщает все существующие нечеткие операции, которые можно получить с помощью генераторов данного типа.

Цель статьи заключается в представлении результатов, касающихся композиции аддитивных генераторов из класса $L F A G$. В разделе 1 представлены общие теоретические сведения о дробно-линейных функциях. Сформулированы теоремы о необходимых и достаточных условиях существования треугольной нормы и конормы для возрастающего и убывающего генераторов в форме $L F A G$. В разделе 2 представлен ряд утверждений, касающихся композиции $L F A G$-генераторов, определены соответствующие треугольные нормы и конормы.

\section{1. МАТЕРИАЛЫ И МЕТОДЫ}

\section{1. Общие сведения о дробно-линейных функциях \\ Функция $\varphi(x)=\frac{a x+b}{c x+d}$, где $a, b, c, d-$ кон-} станты, удовлетворяющие условию $a d-d c \neq 0$, называется дробно-линейной функииец̆, а осуществляемое ею отображение $\varphi$ - дробно-линейным отображением.

Функции $\varphi$ соответствует матрица коэффициентов вида $M_{\varphi}=\left(\begin{array}{ll}a & b \\ c & d\end{array}\right)$.

Под тождественной функцией понимается функция вида $E(x)=x$, при этом $M_{E}=\left(\begin{array}{ll}1 & 0 \\ 0 & 1\end{array}\right)$. Соответствующее отображение называется тождественным.

Для любой дробно-линейной функции $\varphi$, удовлетворяющей условию $a d-d c \neq 0$, можно определить обратную дробно-линейную функцию

$$
\varphi^{-1}(x)=\frac{d x-b}{-c x+a},
$$

для которой также выполняется условие $a d-d c \neq 0$, при этом $M_{\varphi^{-1}}=(a d-b c)\left(M_{\varphi}\right)^{-1}$.
Композищией дробно-линейных функций $\varphi$ и $\psi$ называется функция, получаемая последовательным применением к $x$ сначала отображения $\psi$, а затем $\varphi$, т. е. $(\varphi \circ \psi)(x)=\varphi(\psi(x))$.

Пусть $\varphi(x)=\frac{a x+b}{c x+d}$ и $\psi(x)=\frac{m x+n}{p x+t}-$ дробно-линейные функции, для которых $a d-b c \neq 0$ и $m t-p n \neq 0$ соответственно, тогда $(\varphi \circ \psi)(x)=\varphi(\psi(x))=\frac{(a m+b p) x+(a n+b t)}{(c m+d p) x+(c n+d t)}$.

Таким образом, композиция дробно-линейных функций является дробно-линейной функцией, а, следовательно, множество всех дробно-линейных функций замкнуто относительно операции композиции. Последовательное выполнение произвольного числа дробно-линейных отображений также сводится к дробно-линейному отображению.

Поставим в соответствие функции $\varphi(x)=\frac{a x+b}{c x+d}$ матрицу коэффициентов вида $M_{\varphi}=\left(\begin{array}{ll}a & b \\ c & d\end{array}\right)$, а функции $\psi(x)=\frac{m x+n}{p x+t}-$ матрицу $M_{\psi}=\left(\begin{array}{ll}m & n \\ p & t\end{array}\right)$, тогда матрица

$$
M_{\varphi} \cdot M_{\psi}=\left(\begin{array}{ll}
a m+b p & a n+b t \\
c m+d p & c n+d t
\end{array}\right)
$$

определяет коэффициенты дробно-линейной функции $(\varphi \circ \psi)$, что соответствует формуле (4).

Так как умножение матриц в общем случае не является коммутативной операцией, то и композиция дробно-линейных функций не коммутативна. С другой стороны, легко проверить, что композиция является ассоциативной операцией. Множество всех дробно-линейных функций с коэффициентами, удовлетворяющими условию $a d-d c \neq 0$, образует группу относительно операции композиции.

В [9] доказано, что аддитивный генератор в форме дробно-линейной функции $\varphi(x)=\frac{a x+b}{c x+d}$ порождает коммутативную и ассоциативную операцию $F$ вида (1) с коэффициентами

$$
\begin{gathered}
a_{0}=b d^{2}, a_{1}=a d^{2}, a_{2}=2 a d c-b c^{2}, \\
b_{0}=a d^{2}-2 b c d, b_{1}=-b c^{2}, b_{2}=-a c^{2} .
\end{gathered}
$$




\section{Композиция генераторов нечетких операций}

1.2. Генераторы в форме дробно-линейных функций [11]

Установлено, что возрастающий генератор $L F A G$ имеет вид

$$
\varphi_{\uparrow}(x)=\frac{m x}{r x+l},
$$

причем $\varphi_{\uparrow}(0)=0$, где $m>0, l \geq 0$.

Заметим, что, формула (6) отличается от константы, если $l \neq 0, r=0$ или $l \neq 0, r \neq 0$.

При $r=0$ из (6) получим функцию $\varphi_{\uparrow}(x)=\frac{m}{l} x$. Так как $m>0, l>0$, то $\frac{m}{l}>0$, и данная функция возрастает на $[0,1]$. Отбрасывая положительную константу, получим известный аддитивный генератор $s_{M}$ (табл. 1). Рассмотрим второй случай. Функция (6) имеет вертикальную асимптоту $x=-\frac{l}{r}$ и горизонтальную асимптоту $y=\frac{m}{r}$. Установлено, что данная функция является непрерывной и возрастающей на $[0,1]$ при выполнении одного из следующих условий, определяющих положение асимптот:

$$
\text { a) }\left\{\begin{array} { l } 
{ - \frac { l } { r } < 0 , } \\
{ \frac { m } { r } > 0 ; }
\end{array} \quad \text { b) } \left\{\begin{array}{l}
-\frac{l}{r} \geq 1, \\
\frac{m}{r}<0 .
\end{array}\right.\right.
$$

Анализ этих условий позволяет сформулировать следующее

Утверждение 1. Функция $\varphi_{\uparrow}(x)=\frac{m x}{r x+l}$, где $m>0$, является возрастающим генератором при выполнении одного из следующих условий: а) $l, r>0$ или b) $r<0, l \in[-r, \infty)$.

Разделив числитель и знаменатель функции (6) на $l$, а затем отбрасывая положительную константу $\frac{m}{l}$, получим функцию вида

$$
s_{\rho}(x)=\frac{x}{\rho x+1},
$$

где $x \neq-\frac{1}{\rho}, \rho=\frac{r}{l}$.

Имеет место следующее

Утверждение 2. Треугольная конорма представима в виде

$$
S_{\rho}(x, y)=\min \left\{1, \frac{x+y+2 \rho x y}{1-\rho^{2} x y}\right\}
$$

тогда и только тогда, когда возрастающий генератор имеет вид (7), где $\rho \in(-1,1]$.
На рис. 1 представлены графики функций $s_{\rho}$ и $S_{\rho}$. Видно, что при увеличении $\rho$ поверхность $S_{\rho}$ все более прижимается к верхней грани единичного куба.

Аналогичные результаты имеют место для убывающего генератора, который имеет вид

$$
\varphi_{\downarrow}(x)=\frac{u(x-1)}{v x+w},
$$

где $x \neq-\frac{w}{v}, u<0$ и $v+w>0$.

Заметим, что условие $\varphi_{\downarrow}(1)=0$ выполняется, при этом целесообразно рассматривать следующие случаи, при которых функция (9) имеет смысл:

a) $v=0, w \neq 0$;

b) $v \neq 0, w=0$;

c) $v \neq 0, w \neq 0$.

В случае а) в рамках предположения $w>0$ имеем положительную константу $\left(-\frac{u}{w}\right)$, отбрасывая которую, получим известный генеpaтор $t_{L}$ (табл. 1). В случае b) при $v>0$ также имеем положительную константу $\left(-\frac{u}{v}\right)$. Отбрасывая ее, получим известный генератор $t_{0}$ (табл. 1). Анализ случая с) позволил сформу-

лировать следующее где $x \neq-\frac{w}{v}$, при $u<0$ является убывающим генератором, если $w \geq 0$ и $v \in(-w, \infty)$.

Разделив числитель и знаменатель функции (9) на $w$ и отбрасывая положительный множитель $\left(-\frac{u}{w}\right)$, перейдем к функции вида

$$
t_{\tau}(x)=\frac{1-x}{1+\frac{1}{\tau} x},
$$

где $\tau=\frac{w}{v}$.

Имеет место следующее

Утверждение 4. Треугольная норма представима в виде

$T_{\tau}(x, y)=\max \left\{0, \frac{\tau^{2}(x+y-1)+(1+2 \tau) x y}{\left(\tau^{2}+2 \tau\right)+(x+y-x y)}\right\}$

тогда и только тогда, когда убывающий генератор имеет вид (10), где $\tau \in(-\infty,-2) \cup(0, \infty)$.

На рис. 2 представлены графики функций $t_{\tau}$ и $T_{\tau}$ при различных значениях параметра $\tau$. 

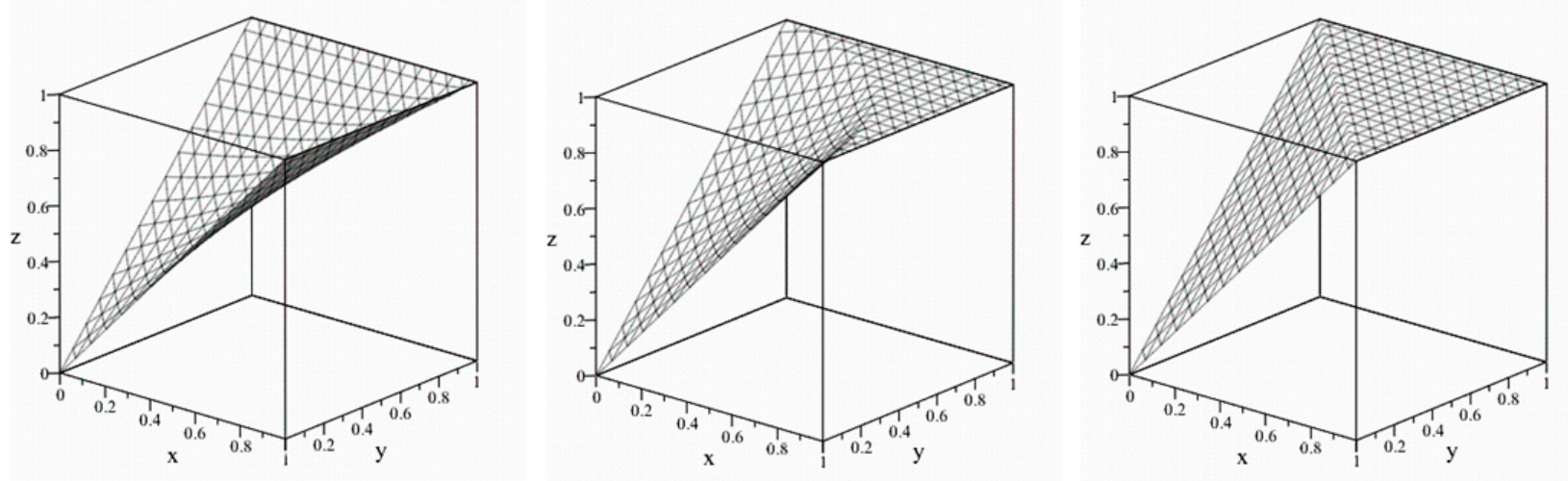

$$
\rho=-1
$$
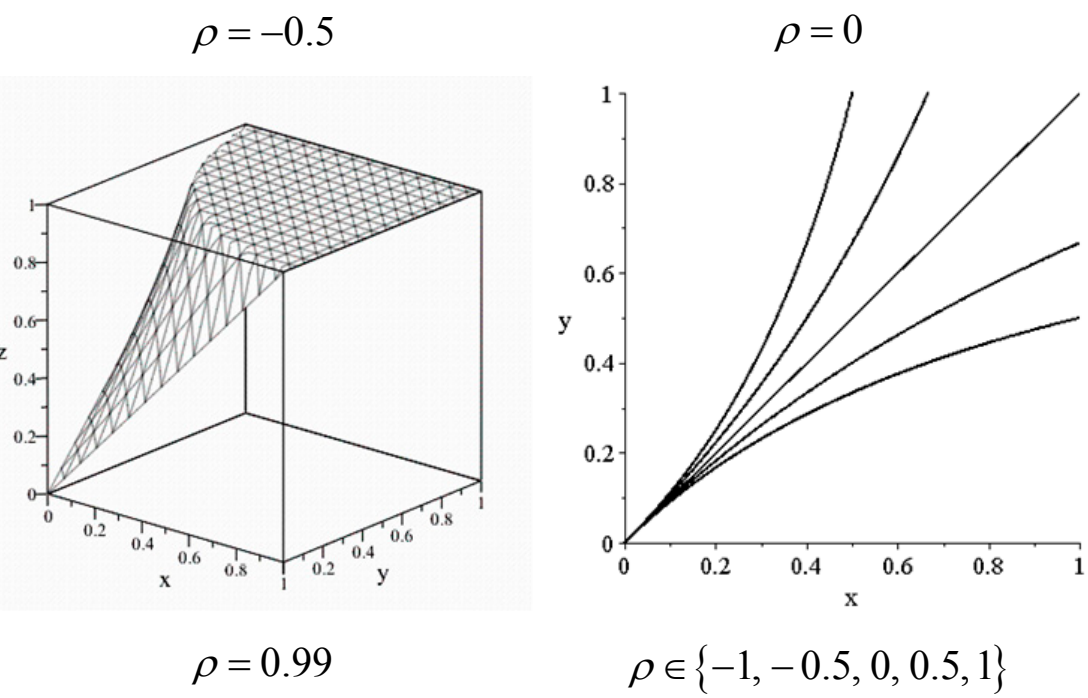

$\rho=0.5$

$\rho=0.99$

$$
\rho \in\{-1,-0.5,0,0.5,1\}
$$

Рис. 1. Графики функиий $s_{\rho}$ и $S_{\rho}$ при различных значениях параметра $\rho$

Заметим, что графики функций для $|\tau|>2$ совпадают, причем даже при существенном увеличении $\tau$ поверхность меняется очень медленно. Для $\tau=0$ операция проектирования не требуется.

В [11] определены условия, при выполнении которых треугольная конорма (8) и треугольная норма (11) являются двойственными (в смысле закона де Моргана) относительно операции стандартного отрицания $1-x$. Также определены все взаимосвязи с существующими классами нечетких операций, для которых аддитивный генератор представляется в форме дробно-линейной функции.

\section{2. РЕЗУЛЬТАТЫ И ИХ ОБСУЖДЕНИЕ}

Утверждение 5. Пусть $\varphi_{\uparrow}(x)=\frac{m_{1} x}{r_{1} x+l_{1}}$ $\left(x \neq-\frac{l_{1}}{r_{1}}\right)$ и $\psi_{\uparrow}(x)=\frac{m_{2} x}{r_{2} x+l_{2}}\left(x \neq-\frac{l_{2}}{r_{2}}\right)-$ возрастающие генераторы, параметры которых удовлетворяют следующим условиям: $m_{1}, m_{2}>0 ; \quad r_{1}>0, l_{1}>0$ или $\quad r_{1}<0, l_{1} \geq-r_{1} ;$ $r_{2}>0, l_{2}>0$ или $r_{2}<0, l_{2} \geq-r_{2}$. Тогда при $r_{1} m_{2}+l_{1} r_{2} \geq-l_{1} l_{2}$ их композиция $\varphi_{\uparrow} \circ \psi_{\uparrow}$, определяемая формулой

$$
\begin{gathered}
\left(\varphi_{\uparrow} \circ \psi_{\uparrow}\right)(x)=\varphi_{\uparrow}\left(\psi_{\uparrow}(x)\right)= \\
=\frac{m_{1} m_{2} x}{\left(r_{1} m_{2}+l_{1} r_{2}\right) x+l_{1} l_{2}},
\end{gathered}
$$

где $x \neq-\frac{l_{1} l_{2}}{r_{1} m_{2}+l_{1} r_{2}}$, также является возрастающим генератором. Если также выполняется условие $r_{1} m_{2}+l_{1} r_{2} \leq l_{1} l_{2}$, то существует треугольная конорма вида 


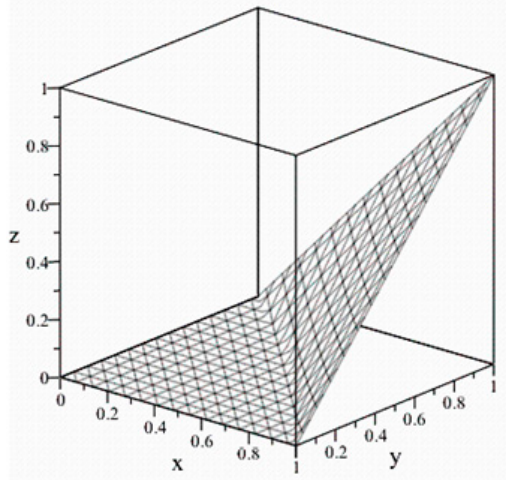

$\tau=-10$

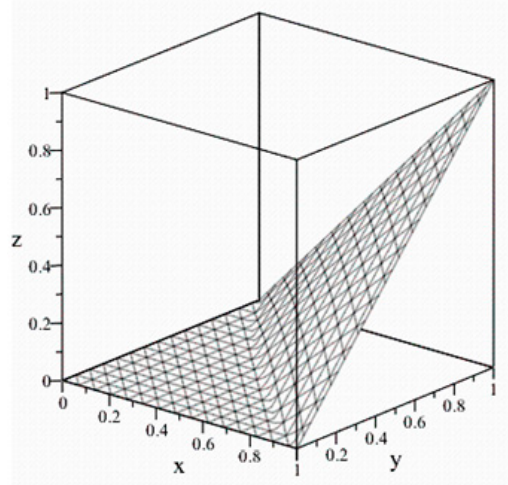

$\tau=5$

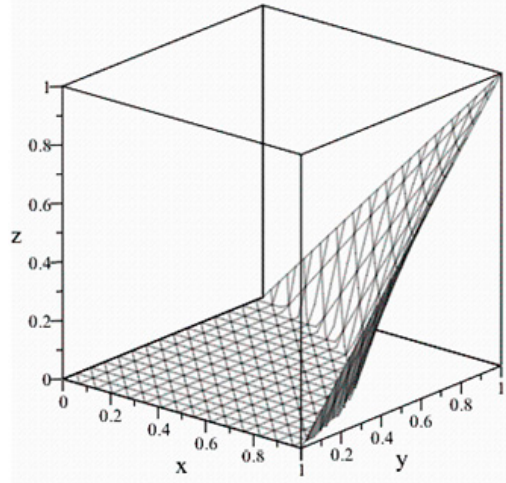

$\tau=-2$

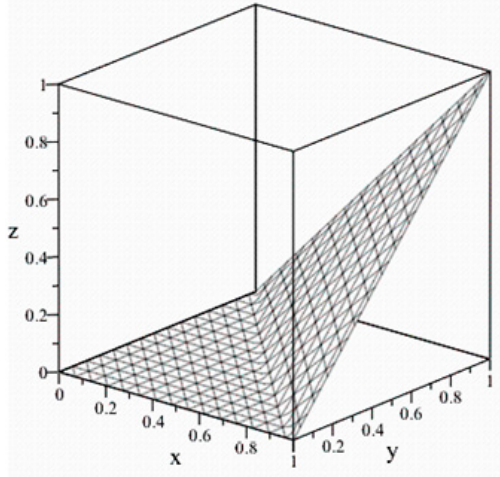

$\tau=10$

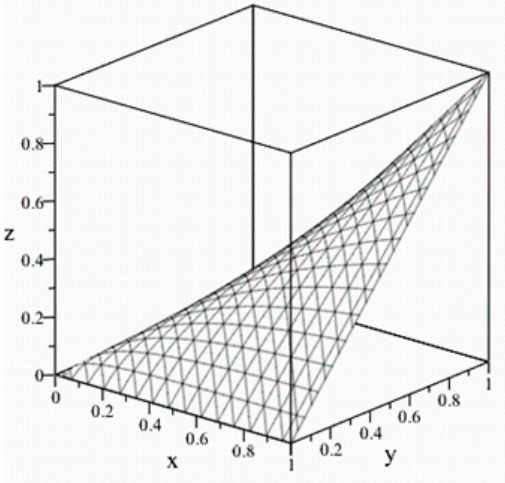

$\tau=0$

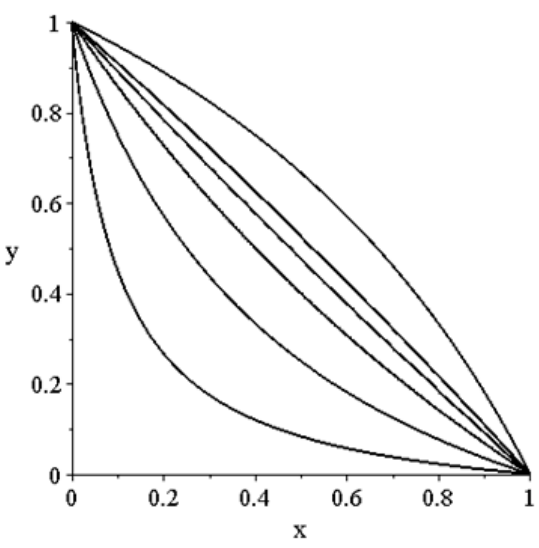

$\tau \in\{-0.5,-2,-10,10,2,0.5,0.1\}$

Рис. 2. Графики функций $t_{\tau}$ u $T_{\tau}$ при различных значениях параметра $\tau$

$$
\begin{aligned}
& S_{\varphi_{\uparrow} \circ \psi_{\uparrow}}(x, y)=S_{\left(l_{1}, r_{i} ; l_{2}, r_{2}\right)}(x, y)= \\
= & \min \left\{\begin{array}{c}
x+y+2 \frac{r_{1} m_{2}+l_{1} r_{2}}{l_{1} l_{2}} x y \\
1-\left(\frac{r_{1}+l_{1} r_{2}}{l_{1} l_{2}}\right)^{2} x y
\end{array}\right\} .
\end{aligned}
$$

Доказательство. По формуле (4) составим композицию $\quad\left(\varphi_{\uparrow} \circ \psi_{\uparrow}\right)(x)=\varphi_{\uparrow}\left(\psi_{\uparrow}(x)\right)=$ $=\frac{m_{1} m_{2} x}{\left(r_{1} m_{2}+l_{1} r_{2}\right) x+l_{1} l_{2}}$. Известно, что композиция возрастающих функций есть возрастающая функция. Кроме того, условие $\left(\varphi_{\uparrow} \circ \psi_{\uparrow}\right)(0)=0$ выполняется. Определим, при каких ограничениях на коэффициенты функция (12) является непрерывной. Функция (12) имеет вертикальную асимптоту $x=-\frac{l_{1} l_{2}}{r_{1} m_{2}+l_{1} r_{2}}$ и горизонтальную асимптоту $y=\frac{m_{1} m_{2}}{r_{1} m_{2}+l_{1} r_{2}}$. Чтобы на $[0,1]$ возрастала правая ветвь графика функции $\varphi_{\uparrow} \circ \psi_{\uparrow}$, должны выполняться следующие условия: $-\frac{l_{1} l_{2}}{r_{1} m_{2}+l_{1} r_{2}}<0 \quad$ и $\frac{m_{1} m_{2}}{r_{1} m_{2}+l_{1} r_{2}}>0$. Так как $m_{1} m_{2}>0, l_{1} l_{2}>0$, то получим неравенство $r_{1} m_{2}+l_{1} r_{2}>0$. Чтобы на $[0,1]$ возрастала левая ветвь графика функции $\varphi_{\uparrow} \circ \psi_{\uparrow}$, должны выполняться условия: $\quad-\frac{l_{1} l_{2}}{r_{1} m_{2}+l_{1} r_{2}} \geq 1 \quad$ и $\frac{m_{1} m_{2}}{r_{1} m_{2}+l_{1} r_{2}}<0$. В этом случае из второго неравенства следует, что $r_{1} m_{2}+l_{1} r_{2}<0$, тогда с учетом первого неравенства имеем $-l_{1} l_{2} \leq r_{1} m_{2}+l_{1} r_{2}<0$. Объединяя результаты, получим, что при $r_{1} m_{2}+l_{1} r_{2} \geq-l_{1} l_{2}$ композиция (12) является возрастающим генератором. Если ввести обозначение $\rho=\frac{r_{1} m_{2}+l_{1} r_{2}}{l_{1} l_{2}}$ и 


\section{Т. М. Леденева}

отбросить в (12) положительную константу $\frac{m_{1} m_{2}}{l_{1} l_{2}}$, то получим семейство генераторов $s_{\rho}$. $\mathrm{B}$ соответствии с утверждением 2 имеем $\rho \in(-1,1]$, а, следовательно, получим неравенство $-1<\frac{r_{1} m_{2}+l_{1} r_{2}}{l_{1} l_{2}} \leq 1$, которое равносильно неравенству $-l_{1} l_{2}<r_{1} m_{2}+l_{1} r_{2} \leq l_{1} l_{2}$. Tреугольная конорма $S_{\varphi_{\uparrow} \nsim \uparrow}$ может быть получена как на основе формул (5), так и на основе утверждения (3) с учетом определения параметра $\rho$.

Пример 1. Рассмотрим возрастающие генераторы $\varphi(x)=\frac{2 x}{3 x+1}$ и $\psi(x)=\frac{x}{2-x}$. Здесь $m_{1}=2, r_{1}=3, l_{1}=1 ; m_{2}=1, r_{2}=-1, l_{2}=2$. Подставляя значения коэффициентов, получим верное неравенство $r_{1} m_{2}+l_{1} r_{2} \geq-l_{1} l_{2}(2>-2)$, поэтому композиция $\left(\varphi_{\uparrow} \circ \psi_{\uparrow}\right)(x)=$ $=\varphi_{\uparrow}\left(\psi_{\uparrow}(x)\right)=\frac{x}{x+1}$ является возрастающим генератором. По формуле (8) для $\rho=2$ найдем соответствующую треугольную конорму $S_{\varphi_{\uparrow} \sim \uparrow}(x, y)=\min \left\{1, \frac{x+y+2 x y}{1-x y}\right\}$. На рис. 3 представлены графики генераторов $\varphi_{\uparrow}, \psi_{\uparrow}$, $\varphi_{\uparrow} \circ \psi_{\uparrow}$ и поверхность $S_{\varphi_{\uparrow} \circ \uparrow}$.

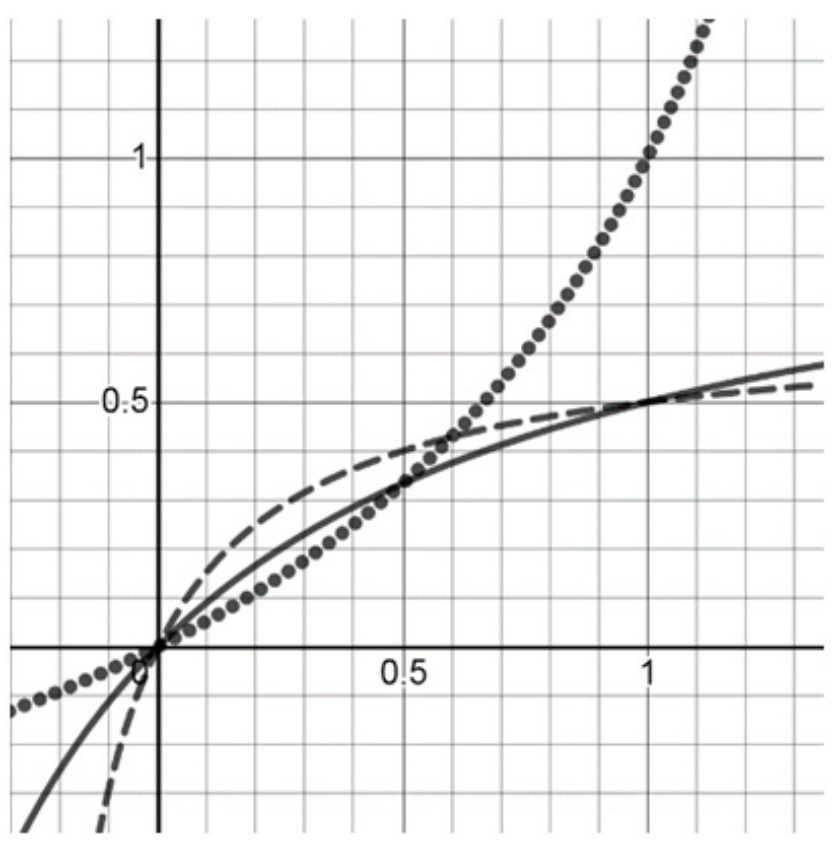

а) Грабик $\varphi_{\uparrow} \circ \psi_{\uparrow}$ изображен сплошной линией
Утверждение 6. Пусть $\varphi_{\downarrow}(x)=\frac{u_{1}(x-1)}{v_{1} x+w_{1}}$ $\left(x \neq-\frac{w_{1}}{v_{1}}\right)$ - убывающий генератор при $u_{1}<0$ и выполнении одного из условий а) $w_{1}>0$, $v_{1} \in\left(-w_{1}, 0\right) \quad$ или $\quad$ b) $\quad v_{1}>0, \quad w_{1} \geq 0$; $\psi_{\downarrow}(x)=\frac{u_{2}(x-1)}{v_{2} x+w_{2}}\left(x \neq-\frac{w_{2}}{v_{2}}\right)-$ убывающий генератор при $u_{2}<0$ и выполнении одного из условий с) $w_{2}>0, v_{2} \in\left(-w_{2}, 0\right)$ или d) $v_{2}>0$, $w_{2} \geq 0$. Тогда при $u_{2}=-w_{2}$ их композиция

$$
\left(\varphi_{\downarrow} \circ \psi_{\downarrow}\right)(x)=\frac{-u_{1}\left(w_{2}+v_{2}\right) x}{\left(v_{2} w_{1}-v_{1} w_{2}\right) x+w_{2}\left(v_{1}+w_{1}\right)},
$$

где $x \neq-\frac{w_{2}\left(v_{1}+w_{1}\right)}{v_{2} w_{1}-v_{1} w_{2}}$, является возрастающим генератором. Если, кроме того, $\frac{v_{2}}{w_{2}}-1 \leq 2 \frac{v_{1}}{w_{1}}$, то существует треугольная конорма вида

$$
S_{\varphi_{\downarrow} \diamond \downarrow \downarrow}(x, y)=\min \left\{1, \frac{P^{2}(x+y)+2 P Q x y}{P^{2}-Q^{2} x y}\right\},
$$

где $P=w_{2}\left(w_{1}-v_{1}\right), Q=v_{2} w_{1}-v_{1} w_{2}$.

Доказательство. На основе (4) составим композицию

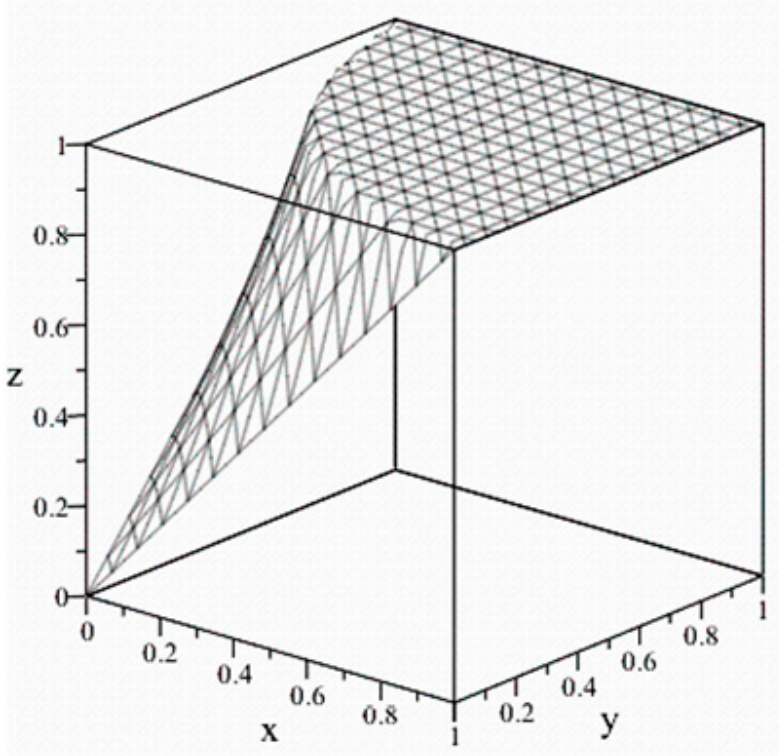

b) Треугольная конорма $S_{\varphi_{\uparrow} \propto \uparrow}$

Pис. 3. Графоки бункциц $\varphi_{\uparrow}, \psi_{\uparrow}, \varphi_{\uparrow} \circ \psi_{\uparrow} u S_{\varphi_{\uparrow} \circ \psi_{\uparrow}}$ 
$\left(\varphi_{\downarrow} \circ \psi_{\downarrow}\right)(x)=\frac{\left(u_{1} u_{2}-u_{1} v_{2}\right) x+\left(-u_{1} u_{2}-u_{1} w_{2}\right)}{\left(v_{1} u_{2}+v_{2} w_{1}\right) x+\left(-v_{1} u_{2}+w_{1} w_{2}\right)}$.

Известно, что композиция убывающих функций есть возрастающая функция, тогда в (16) имеет место равенство $u_{2}=-w_{2}$. Сделав замену в (16), получим (14). Чтобы функция (14) была возрастающим генератором, должны выполняться следующие условия из утверждения 1: $-u_{1}\left(w_{2}+v_{2}\right)>0, w_{2}\left(v_{1}+w_{1}\right)>0$, $v_{2} w_{1}-v_{1} w_{2} \geq-w_{2}\left(v_{1}+w_{1}\right)$. Анализ показывает, что при имеющихся ограничениях на параметры генераторов $\varphi_{\downarrow}$ и $\psi_{\downarrow}$ данные условия выполняются. Таким образом, если $u_{2}=-w_{2}$, то композиция $\varphi_{\downarrow} \circ \psi_{\downarrow}$ убывающих генераторов есть возрастающий генератор. Разделив числитель и знаменатель на $w_{2}\left(v_{1}+w_{1}\right)$ и отбросив положительный множитель $\frac{-u_{1}\left(w_{2}+v_{2}\right)}{w_{2}\left(v_{1}+w_{1}\right)}$, приведем генератор (14) к виду $s_{\rho}$, где $\rho=\frac{v_{2} w_{1}-v_{1} w_{2}}{w_{2}\left(w_{1}-v_{1}\right)}$. В соответствии с утверждением 2 должно выполняться неравенство $-1<\frac{v_{2} w_{1}-v_{1} w_{2}}{w_{2}\left(w_{1}-v_{1}\right)} \leq 1$, которое равносильно следующему неравенству $-w_{2}\left(w_{1}-v_{1}\right) \leq$ $\leq v_{2} w_{1}-v_{1} w_{2} \leq w_{2}\left(w_{1}-v_{1}\right)$. Из полученного не-

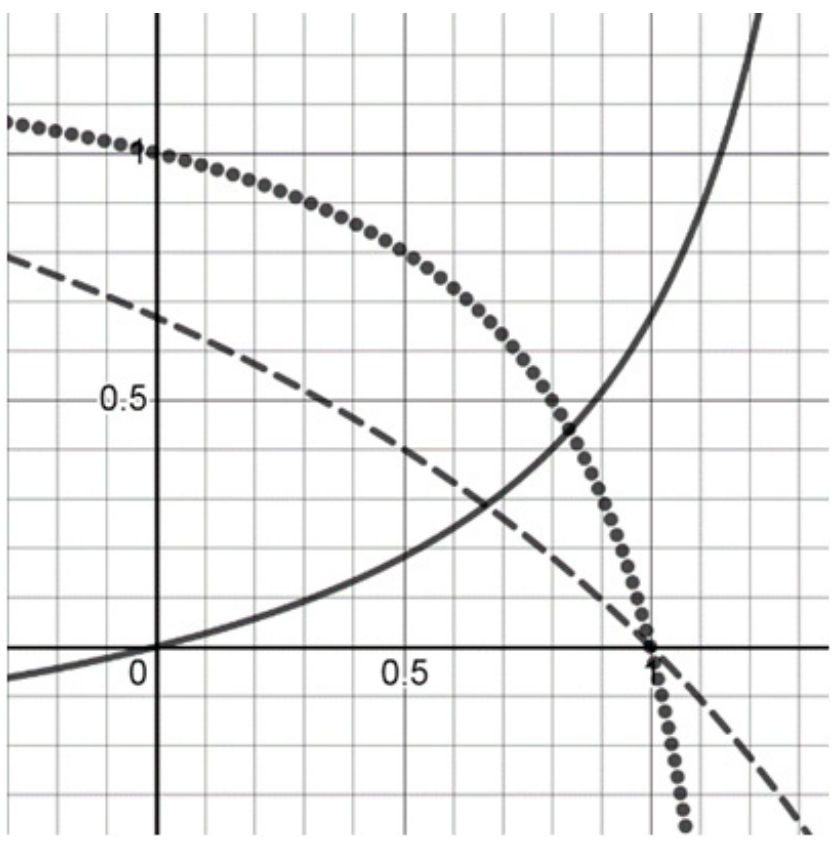

а) Грабик $\varphi_{\downarrow} \circ \psi_{\downarrow}$ изображен сплошной линией равенства имеем следующее ограничение на параметры: $\frac{v_{2}}{w_{2}}-1 \leq 2 \frac{v_{1}}{w_{1}}$.

Пример 2. Рассмотрим убывающие генераторы $\varphi_{\downarrow}(x)=\frac{-2(x-1)}{-x+3}$ и $\psi_{\downarrow}(x)=\frac{-4(x-1)}{-3 x+4}$. Здесь $u_{1}=-2, v_{1}=-1, w_{1}=-3, u_{2}=-4, v_{2}=-3$, $w_{2}=4$. Заметим, что $u_{2}=-w_{2}=-4$, поэтому композиция $\left(\varphi_{\downarrow} \circ \psi_{\downarrow}\right)(x)=\frac{2 x}{-5 x+8}$ является возрастающим генератором. На рис. 4а) представлены графики $\varphi_{\downarrow}, \psi_{\downarrow}, \varphi_{\downarrow} \circ \psi_{\downarrow}$. Найдем $\frac{v_{2}}{w_{2}}=-\frac{3}{4}, \frac{v_{1}}{w_{1}}=-\frac{1}{3}$. Неравенство $\frac{v_{2}}{w_{2}}-1 \leq 2 \frac{v_{1}}{w_{1}}-$ $-\frac{3}{4}-1 \leq 2 \cdot\left(-\frac{1}{3}\right)$ истинно, поэтому треугольная норма существует и имеет вид $S(x, y)=\frac{x+y-5 / 8 x y}{1-25 / 256^{x y}}($ рис. $\left.4 \mathrm{~b})\right)$.

Утверждение $\quad$ 7. Пусть $\quad \varphi_{\uparrow}(x)=\frac{m x}{r x+l}$ $\left(x \neq-\frac{l}{r}\right)$ - возрастающий генератор, где $m>0, \quad l>0, \quad r \in(-l, 0) \cup(0, \infty)$, $\varphi_{\downarrow}(x)=\frac{u(x-1)}{v x+w}\left(x \neq \frac{w}{v}\right)$ - убывающий гене-

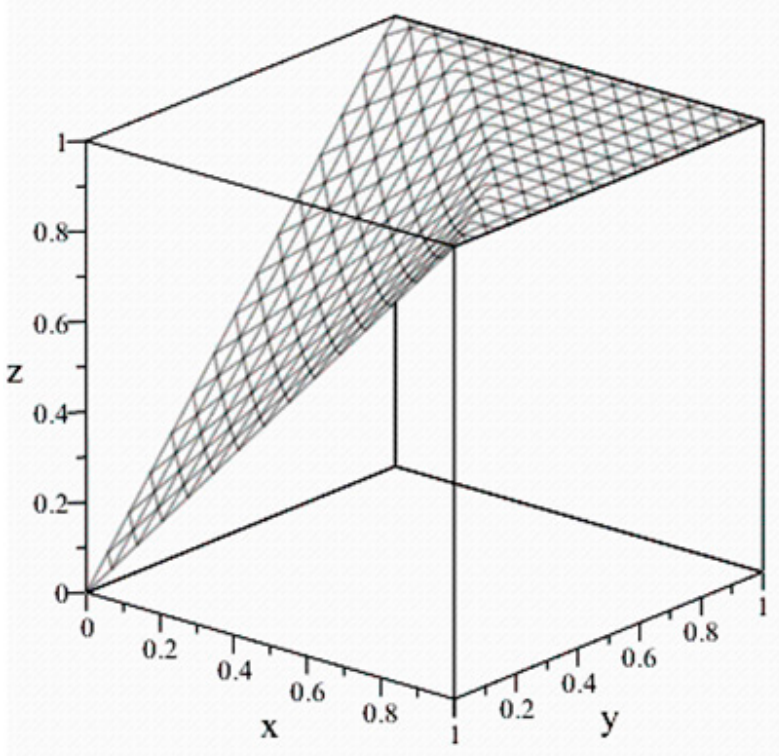

b) Треугольная конорма, соответствующая $S_{\varphi_{\downarrow} \diamond \psi_{\downarrow}}$ Pис. 4. Графоки функциц $\varphi_{\downarrow}, \psi_{\downarrow}, \varphi_{\downarrow} \circ \psi_{\downarrow} u S_{\varphi_{\downarrow} \diamond \downarrow}$ 


\section{T. М. Леденева}

ратор, где $u<0, \quad w>0, \quad v \in(-w, 0) \cup(0, \infty)$, тогда их композиция

$$
\left(\varphi_{\uparrow} \circ \varphi_{\downarrow}\right)(x)=\frac{m u(x-1)}{(u r+v l) x+(-u r+w l)},
$$

где $x \neq-\frac{-u r+w l}{u r+v l}$, является убывающим генератором, $u r+v l$ если $-u r+w l>0 \quad$ и $u r+v l \in(u r-w l, 0) \cup(0, \infty)$. Если при этом отрицательные значения $(u r+v l)$ принадлежат только интервалу $\left(\frac{u r-w l}{2}, 0\right)$, то существует треугольная норма вида

$$
\begin{gathered}
T_{\varphi_{\uparrow} \circ \varphi_{\downarrow}}(x, y)= \\
=\max \left\{0, \frac{P^{2}(x+y-1)+\left(Q^{2}+2 P Q\right) x y}{\left(P^{2}+2 P Q\right)+Q^{2}(x+y-x y)}\right\},
\end{gathered}
$$

где $P=-u r+w l, Q=u r+v l$.

Доказательство. На основе (4) найдем композицию, которая имеет вид (17) и согласуется с представлением убывающего генератора. Заметим, $m u<0$ по условию. В соответствии с утверждением 3 для коэффициентов функции (17) должны выполняться следующие ограничения на параметры: $-u r+w l \geq 0$ и $u r+v l \in(-(-u r+w l), \infty)$. Разделим числитель и знаменатель $(17)$ на $(-u r+w l)$. Отбросив положительную константу $\frac{-m u}{-u r+w l}$, можно заметить, что замена $\tau=\frac{-u r+w l}{u r+v l}$ позволяет перейти от (17) к генератору $t_{\tau}$. Следовательно, можно использовать утверждение 4 для нахождения ограничений на параметры (17). Согласно им, должно выполняться одно из следующих неравенств: $\frac{-u r+w l}{u r+v l}>0$ или $\frac{-u r+w l}{u r+v l}<-2$. Первое неравенство имеет место, если $u r+v l>0$. Из второго неравенства при $u r+v l<0$ получим, что $u r+v l>-\left(\frac{-u r+w l}{2}\right)$. Окончательно, получим, что если $u r+v l \in\left(-\left(\frac{-u r+w l}{2}\right), 0\right) \cup(0, \infty)$, то существует треугольная норма в виде (18), которая соответствует генератору (17).
Пример 3. Рассмотрим возрастающий генератор $\varphi_{\uparrow}(x)=\frac{x}{4-3 x}$ и убывающий генератор $\varphi_{\downarrow}(x)=\frac{1-x}{3-2 x}$. Здесь $m=1, r=-3, l=4$, $u=-1, v=-2, w=3$. Вычислим $-u r+w l=9$, $u r+v l=-5$. Заметим, что $-5 \in(-9,0)$, поэтому композиция $\left(\varphi_{\uparrow} \circ \varphi_{\downarrow}\right)(x)=\frac{1-x}{9-5 x}$ является убывающим генератором. Но треугольной нормы не существует, так как $\left(\frac{u r-w l}{2}, 0\right)=$ $=(-4.5,0)$ и $-5 \in(-4.5,0)$.

Утверждение 8. Пусть $\varphi_{\downarrow}(x)=\frac{u(x-1)}{v x+w}$ $\left(x \neq-\frac{w}{v}\right)$ - убывающий генератор, где $u<0$, $w>0, v \in(-w, \infty), \varphi_{\uparrow}(x)=\frac{m x}{r x+l}\left(x \neq-\frac{l}{r}\right)-$ возрастающий генератор, где $m>0, l>0$, $r \in(-l, \infty)$, тогда их композиция

$$
\left(\varphi_{\downarrow} \circ \varphi_{\uparrow}\right)(x)=\frac{u l(x-1)}{(m v+w r) x+w l}
$$

является убывающим генератором, если $l+r=m . \quad$ Если, кроме того, $v+\frac{r}{m} w \in(-(v+w), 0) \cup(0, \infty)$, то существует треугольная норма вида

$$
\begin{gathered}
T_{\varphi_{\downarrow} \circ \varphi_{\uparrow}}(x, y)= \\
=\max \left\{0, \frac{P^{2}(x+y-1)+\left(Q^{2}+2 P Q\right) x y}{\left(P^{2}+2 P Q\right)+Q^{2}(x+y-x y)}\right\},
\end{gathered}
$$

где $P=w-w r, Q=v+w r$.

Доказательство. На основе (7) найдем композицию

$$
\left(\varphi_{\downarrow} \circ \varphi_{\uparrow}\right)(x)=\frac{u(m-r) x-u l}{(m v+w r) x+w l} .
$$

Заметим, что композиция убывающей и возрастающей функций есть убывающая функция. Если в (21) положить $m-r=l$, то функция приводится к виду (19) и может рассматриваться как убывающий генератор. Здесь $u l<0, w l>0$. В соответствии с утверждением 3 должно выполняться неравенство: $m v+w r \geq-w l$. Легко показать, что оно эквивалентно следующему условию $(v+w)(l+r) \geq 0$, 
которое всегда выполняется при существующих ограничениях на коэффициенты.

Найдем треугольную норму, которая соответствует данному генератору. Если разделить числитель и знаменатель (19) на $w l$, отбросить положительную константу $\left(-\frac{u}{w}\right)$, то получим генератор $t_{\tau}$, где $\tau=\frac{w l}{m v+w r}$. В соответствии с утверждение 4 для существования треугольной нормы должно выполняться ограничение $\frac{w l}{m v+w r} \in(-\infty,-2) \cup(0, \infty)$. Легко показать, что данное ограничение равносильно следующему: $-(v+w)<v+\frac{r}{m} w<0$ или $v+\frac{r}{m} w>0$. Треугольная норма получается из формулы (11) при данном $\tau$.

Пример 4. Рассмотрим генераторы $\varphi_{\uparrow}(x)=\frac{x}{2-x}$ и $\varphi_{\uparrow}(x)=\frac{1-x}{-x+3}$. Здесь $m=1$, $r=-1, l=2, u=-1, v=-1, w=3$. Вычислим $v+w=2, \quad v+\frac{r}{m} w=-4 . \quad$ Так как $l+r=2-1=1=m$, то существует убывающий генератор $\left(\varphi_{\downarrow} \circ \varphi_{\uparrow}\right)(x)=\frac{1-x}{-2 x+3}$. Однако, треугольная норма не существует, так как $v+\frac{r}{m} w=-4 \notin(-2,0) \cup(0, \infty)$.

\section{ЗАКЛЮЧЕНИЕ}

Исследование треугольных норм и конорм в терминах их аддитивных генераторов лежит в основе ряда приложений, среди которых выделим следующие: конструирование различных нечетких алгебр; определение различных видов композиций нечетких отношений и типов транзитивности; определение специальных операций над нечеткими числами на основе принципа обобщения; определение операций агрегирования.

В статье представлены результаты исследования аддитивных генераторов в форме дробно-линейных функций. Известно, что семейство дробно-линейных функций замкнуто относительно операции композиции. В рамках статьи определены ограничения на параметры, при выполнении которых композиции возрастающих и/или убывающих генераторов в форме дробно-линейных функций $L F A G$ также являются генераторами из данного класса. В каждом случае определены соответствующие треугольные нормы и конормы. Установлено, что для данного класса генераторов треугольные нормы и конормы имеют определенную структуру, формула которой в общем случае не сводится к известным семействам нечетких операций, а обобщает их.

\section{СПИСОК ЛИТЕРАТУРЫ}

1. Klement, E. P. Triangular norms. Position paper I: basic analytical and algebraic properties / E. P. Klement, R. Mesiar, E. Pap // Fuzzy Set and Systems, 2004. - № 143. - P. 5-26.

2. Klement, E. P. Triangular norms. Position paper II: general constructions and parameterized families / E. P. Klement, R. Mesiar, E. Pap // Fuzzy Set and Systems, 2004. - № 145. - P. 411438.

3. Klement, E. P. Triangular norms. Position paper III: continuous t-norms / E. P. Klement, R. Mesiar, E. Pap // Fuzzy Set and Systems, 2004. - № 145. - P. 5-26 145(2004) 439-454.

4. Schweizer, B. Probabilistic Metric Spaces / B. Schweizer, A. Sklar. - North-Holland, New York, 1983.

5. Klir, G. J. Fuzzy sets and fuzzy logic: theory and applications / G. J. Klir, Yuan Bo. - Prentice Hall, 1995. - 574 p.

6. Klement, E. P. Problems on triangular norms and related operators / E. P. Klement, R. Mesiar, E. Pap // Fuzzy Set and Systems, 2004. - № 145. P. 471-479.

7. Navara, M. Explicit formulas for generators of triangular norms / M. Navara, M. Petrik, P. Sarkoci // Publ. Math. Debrecen, 2010. № 77/1-2. - P. 171-191.

8. Ouyang, Y. On the construction of boundary weak triangular norms through additive generators / Y. Ouyang // Nonlinear Analysis, 2007. № 66. - P. 125-130. 


\title{
T. М. Леденева
}

9. Леденева, T. M. Некоторые аспекты Conf.Series973(2018), https://doi:10.1088/1742представления нечетких операторов отношением двух многочленов / Т. М. Леденева // Известия ВУЗов. Математика, 1997. - С. 33-40.

10. Ledeneva, T. Analysis of additive generators of fuzzy operations represented by rational functions / T. Ledeneva. - Journal of Physics: 6596/973/1/012037

11. Ledeneva, T. Additive generators of fuzzy operations in the form of linear fractional functions / T. Ledeneva. - Fuzzy Set and Systems (2019), https://doi.org/10.1016/j.fss.2019.03.005

Леденева Т. М. - д-р техн. наук, профессор, заведующий кафедрой вычислительной математики и прикладных информационных технологий, факультет прикладной математики, информатики и механики, Воронежский государственный университет, e-mail: ledeneva-tm@yandex.ru

\section{COMPOSITION OF GENERATORS OF FUZZY OPERATIONS}

\author{
T. M. Ledeneva \\ Voronezh State University
}

\begin{abstract}
Annotation. This article is devoted to the study of additive generators in the form of linear fractional functions. It is shown that compositions of increasing and/or decreasing generators are also generators. For each case, the corresponding triangular norms or conorms are found. It is established that the triangular norms and conorms obtained on the basis of linear fractional functions, as well as dual ones, have the same structure.

Keywords: triangular norms and conorms, decreasing and increasing generators.
\end{abstract}

Ledeneva T. M. - Doctor of Technical Science, Professor, Department of Computational Mathematics and Applied Information Technologies, Faculty of Applied Mathematics, Informatics and Mechanics, Voronezh State University, e-mail: ledeneva-tm@yandex.ru 